\title{
OPINIÓN EXPERTA Y GASTRONOMÍA. LA RECETA PARA EL ÉXITO
}

\author{
Véronique Chossat y Olivier Gergaud *
}

\section{RESUMEN}

Las opiniones de los expertos juegan un papel importante en el mercado gastronómico por las siguientes razones: la información es imperfecta y bastante costosa de adquirir; la calidad en gran parte- es subjetiva y los consumidores necesitan que los expertos la definan. El número de manuales actualmente editados, su éxito (Michelin: 650,000 vendidos; GaultMillau: 200,000 vendidos) y el fuerte nivel de correlación generalmente obtenida entre los precios e índices o clasificaciones ( 0.63 en promedio) para esta clase de actividad ilustran esta influencia. Sin expertos, la oferta y la demanda difícilmente concordarían. Por consiguiente, es relevante identificar los determinantes de estas evaluaciones de calidad y luego estimar su impacto respectivo.

De acuerdo a los expertos, el arte de cocinar es el único determinante que ellos toman en cuenta cuando seleccionan y luego evalúan a los chefs. Para estos, el ambiente o entorno también aparece como un determinante y no el menos importante. ¿Cuál es la mejor estrategia para llegar a ser un chef de "primer nivel"? ¿Conseguiría Alain Ducasse, uno de los más famosos chefs franceses, el mismo nivel en un café al costado de la carretera como en un restaurante de lujo?
Para responder estas interrogantes se estima una ecuación de calidad usando una base de datos original que concierne a 185 chefs franceses líderes, quienes han sido seleccionados en uno de los más famosos manuales franceses: GaultMillau (edición 2000). Los resultados muestran que hay dos estrategias para llegar a ser un "chef de primer nivel", pero que el arte de cocinar prevalece sobre el ambiente o entorno. Esto está de acuerdo con la observación de que algunos restaurantes gastronómicos tienden a invertir en forma excesiva en una ambientación lujosa.

Palabras-clave: Opinión experta, gastronomía, calidad.

\section{INTRODUCCIÓN}

La mayoría de las (pocas) contribuciones teóricas enfocadas en la demanda por restaurantes (Becker, 1991; Banerjee, 1992; Albrecht et al., 2002) ignoran o tratan la calidad como una variable exógena. Sin embargo, como en el caso de muchos otros bienes $y$ servicios culturales (que van de la danza al teatro y de la música al vino) y no-culturales (mercado de valores, carreras de caballos, entre otros), la opinión experta es una determinante crítica de

- Profesor de la Universidad de Reims, Reims, Francia. Agradecemos a los autores que generosamente nos permitieran difundir su estudio en castellano. La versión inglesa del texto fue publicada en Journal of Cultural Economics 27: 127-141, 2003. La traducción estuvo a cargo de Oscar del Águila, del Instituto de Investigaciones de la Escuela Profesional de Turismo y Hotelería de la USMP. 
la demanda para la cocina gourmet ${ }^{1}$. El papel de la opinión experta y la eficiencia de la demanda por ella han sido cuestionados en estudios de vino (Ashenfelter y Jones, 2000) y de actuaciones musicales (Ginsburgh y Van Ours, 2002) $)^{2}$. En todas estas aplicaciones una pregunta principal es: ihasta qué punto las opiniones expertas reflejan los fundamentos subyacentes de valor $y$ hasta qué punto reflejan factores que no están relacionados con los fundamentos? En este artículo estudiamos los determinantes de la calidad de los chefs franceses evaluados por manuales. La pregunta clave que hacemos es: ison estos índices principalmente determinados por la calidad de la comida presentada o es algo más implicado en los fallos de los críticos?

Evaluar la calidad de un restaurante es en sí una tarea difícil que requiere un esfuerzo considerable por dos razones. Primero: el mercado gastronómico es por definición geográficamente disperso, uno necesita desplazarse para consumir, haciendo que adquirir la información resulte bastante costoso. Segundo: la evaluación es principalmente subjetiva, el servicio que aquí estamos considerando consiste mayormente en lo que los economistas llaman experiencia y características crediticias, es decir respectivamente descubiertas, o no, luego de que un producto ha sido consumido (por ejemplo la calidad de los ingredientes, el sabor de la preparación, etc.). Por otro lado, el servicio despeja pocas características de investigación, mientras otras pueden ser comprobadas durante el proceso de búsqueda con anterioridad a la compra, por ejemplo el arreglo o escenario del restaurante ${ }^{3}$. Básicamente, la información acerca de restaurantes y cocineros es largamente imperfecta. Por lo tanto, el problema principal que enfrentan los consumidores de gastronomía es cómo encontrar información útil.
En este artículo analizamos empíricamente cómo los expertos clasifican los restaurantes, cuáles son sus criterios y cuál es el impacto relativo de estos criterios en las ubicaciones finales y notas generales. Este tema es de interés porque los manuales de los expertos en gastronomía no solo tienen gran influencia en los niveles de precio, sino también en la elección del consumidor, y aun tal vez en el estilo de cocinar (clásico, creativo, regional...). Supongamos que la creación esté de moda y lo clásico no. Uno puede esperar que los chefs tiendan a favorecer la creatividad frente a lo clásico para obtener buenas evaluaciones y cobrar más. La primera evidencia empírica del gran papel jugado por los críticos en este mercado es ciertamente el fuerte y significante nexo estadístico generalmente obtenido entre el precio cobrado por un restaurante $y$ las evaluaciones que este recibe de los manuales más influyentes ${ }^{4}$. La segunda evidencia es el hecho de que, en la mayoría de países desarrollados, los manuales son numerosos y variados ${ }^{5}$.

El papel de los expertos es seleccionar entre un amplio rango de chefs a los que ameriten la denominación de "gran chef", es decir de cocinero de alta clase. De acuerdo con Wijnberg (1995) "en la selección del experto, una especial capacidad evaluativa es atribuida al relativamente pequeño grupo de seleccionadores, quienes no son miembros del grupo en el cual el proceso de selección toma lugar" ${ }^{\prime \prime}$. Este procedimiento resulta de la aplicación por cada manual de un patrón específico de criterio de la calidad gastronómica: su "convención de calidad", si nos referimos a la Escuela Francesa de Convenciones (Favereau y Lazega, 2002). Debido a que los manuales gastronómicos son negocios privados en competencia unos con otros y también debido a la necesidad de evaluar la dimensión creativa del servicio,

1 La cocina gourmet o gastronómica puede ser visıa como el segmento más elevado del mercado de catering o restauración. De acuerdo con el Instituto Nacional Francés de Información Económica y Estadística (1999), el mercado de catering está conformado por más de 100,000 negocios, tiene una facturación de 17 billones de euros y anualmente provee más de 2.5 billones de comidas (ver Chossat, 2001 para más detalles).

2 Ver también Ginsburgh (2002) para una visión más global.

3 Los dos conceptos de investigación buena y experiencia buena fueron introducidos por Nelson (1970, 1974). La noción de crédito bueno fue introducida luego por Darby y Karni (1973). Previamente, Lancaster (1966) consideró una comida simple con dos tipos de caracteristicas -nutricional y estética- y una cena como una mezcla de estas dos características, más una adicional, la caracteristica intelectual, conectada al entorno social.

4 En promedio, la correlación simple coeficiente entre precio e indice es 0.63.

5 En Francia una distinción puede ser hecha entre el lider -Michelin $(650,000$ copias vendidas en el año 2000$)$, la más famosa y antigua- y sus principales seguidores, Gaultmillau, Champérard y Botín Courmand.

6 Iniciada entre 1803 y 1812 por el Almanach des Courmands de Grimod de la Reynière, los principios de la selección gastronómica esperaron para la publicación de la guía de Michelin para perpetuar las "étoiles de bonne table" (Michelin stars) en fuerza desde 1933. 
los expertos mantienen sus sistemas de calificación en secreto por razones estratégicas. La Guía Roja (Michelin) se distingue a sí misma como la más discreta; no brinda comentarios con sus estrellas, contrariamente a su competidor más cercano $^{7}$. Botin Gourmand, Champérard y GaultMillau proveen una breve reseña de cada restaurante pero se mantienen extremadamente evasivos acerca de la forma en que los locales son evaluados y clasificados.

En estas guías generalmente se declara que solamente el tipo de cocina (la creatividad, la técnica, la calidad de los ingredientes, etc.) es evaluado y luego premiado. En otras palabras, los elementos del servicio no tienen influencia alguna en las evaluaciones, aun si los comentarios escritos frecuentemente contienen indicaciones acerca del escenario (ambiente, servicio, bodega, etc.). Para el chef, el conjunto del servicio es una estrategia alternativa que puede ser implementada con la esperanza de crecer jerárquicamente. En efecto, ajustar los niveles del entorno con los niveles de cocina parece ser particularmente bien percibido por los expertos.

¿Quién está en lo correcto? ¿Los chefs o los expertos? ¿Acaso los chefs solo tienen una estrategia (el cocinar) para obtener buenas mediciones de los expertos, o es que enfrentan un intercambio entre dos estrategias (el cocinar o el servicio)? ¿Conseguiría Alain Ducasse, uno de los más famosos chefs franceses, el mismo nivel en un café al costado de la carretera como en un restaurante de lujo? ¿Cuáles son los incentivos para un chef de invertir en su entorno? ¿En su estilo de cocinar? ¿Los chefs invierten en su entorno en perjuicio de su cocina? ¿Es eficiente mantener una buena bodega en un restaurante para proveer de buenos vinos a los clientes? ¿Y qué pasa acerca del senvicio convivial? ¿Es la cocina más, menos o tan importante como el entorno del restaurante? Por todas estas razones, llega a ser relevante el preguntarse cuál es la receta para el éxito en la gastronomía francesa.
Para responder a estas preguntas y encontrar esta receta misteriosa, estimamos una ecuación de calidad usando una original base de datos que incluye cerca de 200 chefs franceses líderes seleccionados por GaultMillau (edición 2000). Este manual -el principal reto de Michelin (200,000 copias en el 2000) - ha sido usado por los comentarios detallados que contiene. Contrariamente a la situación de los vinos, para los cuales existe un glosario preciso, en la comida gastronómica no hay un glosario oficial disponible. Y lo que es más, en este análisis debemos tener en cuenta el hecho de que el entorno (cuadro, servicio y bodega) es potencialmente correlacionado con el estilo de cocinar. Este problema endogenético es común en economía, particularmente en economías laborales donde la correlación entre la educación y los salarios no necesariamente quiere decir que el primer factor determine al segundo (ver Card, 1999, para una encuesta).

Este artículo está organizado de la manera siguiente: en la sección 2 se presentan los datos, la sección 3 contiene los resultados empíricos, seguida de algunos puntos concluyentes.

\section{LOS DATOS 8}

La muestra ha sido restringida a una categoría de cocineros principales (los asistentes no son considerados), miembros de The Master Chefs of France Association (edición 1999), cuyos restaurantes están ubicados en Francia metropolitana y que trabajan dentro del circuito comercial del mercado de servicio de comidas. El número total de cocineros incluidos es de 185.

El manual de GaultMillau (CM) selecciona los restaurantes en una escala de rangos, en teoría de 0 a 20, pero en la práctica los grados que son designados (notas) están entre el 11 y el 19. Es útil indicar que hay una fuerte $y$ significativa correlación entre el grado atribuido a un cocinero y la amplitud del comentario (0.84), y que

7 Desde su edición 2000, tres (conas) lineas de comentarios que principalmente se centran en el entomo del restaurante y su historia, se añaden a cada restaurante.

8 Ver el apéndice para las descripciones estadísticas para nuestros dalos. 
la estructura de este es estable con tres partes. La primera concieme exclusivamente al cocinero y a su tipo de cocina, empieza con algunos puntos acerca de la ubicación geográfica y la historia del restaurante, del cocinero y su familia y continúa con una presentación del tipo de cocina que practica (calidad de los platos, la técnica, el conocimiento, profesionalismo, estilo de cocinar, calidad de los ingredientes, etc.). La segunda parte se enfoca en los suplementos extras-usualmente el vino-, mientras que la última parte aborda el servicio y su entorno.

En resumen, la dispersión del grado parece depender de tres grandes criterios 9 :

1. El tipo o estilo de cocina.

2. La bodega.

3. El entorno y el servicio.

\section{La cocina}

\section{- Comentarios generales}

Los comentarios generales introducen al chef $y$ su forma de cocinar con más o menos expresiones laudatorias o loables como, por ejemplo: "él ahora se sienta en lo más alto de la Montaña Olímpica de la Gastronomía" o "un mega chef". Los comentarios pueden también mencionar uno o varios cursos particularmente apreciados o resaltantes ${ }^{10}$. Estas dos variables han sido integradas en una sola para evitar potenciales problemas multiculinarios. Estos superlativos dan una imagen global de cómo un cocinero y su producción son percibidos por los expertos GM (GaultMillau). El número de superlativos es presentado por 4 modelos: SUP 1-5 cuando el total es mayor o igual a 1 y menor que 5, SUP 5-10 cuando el total es mayor o igual a 5 y menor que 10, SUP 10-15 cuando el total es mayor o igual a 10 y menor que 15, SUP-15 cuando el total es 15 o más.

El cocinero y su forma de cocinar pueden, en algunas circunstancias, ser criticados: "falta de madurez" o "una experiencia decepcionante". En este caso el modelo (CRIT) tomará el valor 1 o de otra forma el valor 0 .
En la mayoría de los casos el estilo de cocinar es enfatizado y al respecto han sido usadas tres categorías: cocina regional (terroir en francés) (REG), como en "uno puede oler el aire abrasante de la región rural de Lozére"; cocina creativa (CREA), como en "comida marina reinventada", y cocina clásica (CLAS), como en "platos clásicos que construyeron su éxito". La cuisine de terroir (sic) es cocina regional: bouillabaisse en el sur de Francia, quenelles en Lyon, choucroute en la región Alsace, y así sucesivamente. La creatividad consiste, por ejemplo, en introducir cambios radicales en el tipo de cocina tradicional, o experimentar con nuevas combinaciones de rellenos como pescado y carne en la misma comida. La comida clásica o tradicional señala la reproducción por un chef de los estándares o cánones de la cocina gastrónoma francesa (volaille en vessie, o boeuf bourguignon, por ejemplo). Cuando ningún estilo es sugerido o percibido, el modelo N/ESTILO toma el valor de 1 o de otra forma el valor 0 .

Debido a que un chef frecuentemente combina más de uno de los estilos de cocina mencionados anteriormente, los modelos CLCR, CLRE, RECR, CLRECR también serán incluidos en la ecuación de grado de los expertos. Estos modelos toman el valor de 1 cuando las combinaciones -clásica-creativa, clásica-regional, regionalcreativa o los tres estilos juntos- son mencionados; de otra manera serán todos cero.

\section{- Comentarios técnicos}

El manual también menciona la técnica y el conocimiento del chef, refiriéndose, por ejemplo, a la calidad de las salsas y/o de las preparaciones. Cuando el contenido de los comentarios es positivo, como por ejemplo en: "pechuga de pollo en una delicada salsa chenil", el modelo TECN+ toma el valor de 1 o de otra forma el valor 0 . Por otro lado, cuando este atributo es percibido negativamente, como en "una pechuga de codorniz hojaldre estaba en su lado seco", TECN- toma el valor de 1 o de otra forma el valor 0 .

9 Asumimos que los expenos CM siempre evalúan los restaurantes refiriéndose a unos criterios especificos y establecidos.

10 Cursos criticados son tomados en cuenta en otra categoria, ver debajo. 


\section{- Otros comentarios}

Los modelos $\mathrm{SAB}+$ y SAB- toman el valor de 1, respectivamente, si la combinación de sabores prueba ser un éxito, como en "una combinación maravillosa de sabores y gustos", o un fracaso, como en "los pimientos rojos sobredimensionaron a la ensalada de langosta", y por ende 0.

Algunos puntos concernientes a la delicadeza de las preparaciones (REF): "una ensalada de almejas de la bahía y espárragos verdes"; su simplicidad (SIMP): "extremadamente simples langostinos rostizados a la sartén"; su sutileza (SUT): "un modelo de fineza" o la habilidad artística del chef (ART): "prueba su gran arte" o "sus creaciones son sorprendentes". Además algunos comentarios frecuentemente sugieren una sofisticación positiva (SOF+): "un maravilloso plato sofisticado", o una negativa (SOF-), que es inútil, inclusive dañina para la preparación, como en "demasiada sofisticación en este plato". La generosidad tiene una dimensión ambivalente: puede ser positiva (GEN+) como en "sirve en porciones generosas" o negativa (GEN-) como en una "falta de generosidad". Finalmente, algunas veces el manual enfatiza la armonía perfecta entre todos los platos que componen el menú (MENÚ). Todas estas variables son modelos, que toman el valor 1 cuando los expertos consideran que el tipo de cocina tiene la llamada característica o de otra forma el valor 0 .

\section{- Calidad de los ingredientes}

En tanto que un chef también es evaluado en su capacidad de seleccionar buenos ingredientes, el GM señala el uso de estos, tanto buenos (ING+): "todos los ingredientes son seleccionados como la misma vista de águila", como malos (ING-): "malogrado por frijoles indigestos".

Finalmente creamos varios modelos para el uso por el chef de comidas raras como, por ejemplo, pescado noble (salmón, rodaballo...) (PESC), crustáceos (pescado crudo...) (CRUS), caviar (CAV), foie gras (FOIE) o trufas (TRUF). Para algunos restaurantes puede ser que el manual enfatice "los quesos muy buenos". En este caso, el modelo QUE tomará el valor 1 o de otra forma el valor 0 .

\section{La bodega}

El GM da comentarios generales acerca de los vinos que un cliente puede encontrar en un restaurante en particular. Por esta razón, preferimos otro indicador para la calidad de la bodega: el contenido en el manual de Champérard, donde un restaurante puede tener de 1 a 4 símbolos de muestras de vinos (VINO = 1 a 4), correspondiendo el 4 a una bodega excepcional con muchos buenos vinos.

\section{El entorno y el servicio}

Nuestro análisis del entorno y del servicio está basado en tres principales categorías o indicadores contenidos en diferentes manuales: $G M, \mathrm{Mi}$ chelin y Champérard.

El primero clasifica los restaurantes en tres diferentes categorías: "bistrot, neobistrot, brasserie y auberge (inn)" (AMBGM=1); "tradicional, confortable o aun elegante" (AMBGM $=2)$; $y$ "prestigio, restaurante lujoso" (AMBCM $=3$ ).

Michelin distingue más categorías para el entorno, dándole al restaurante de 1 a 5 "tenedores y cucharas" negros o rojos. En este contexto rojo es mejor que negro y señala un entorno más agradable dentro de cada categoría. En la ecuación de nota de los expertos, la variable F\&S indicará el número de tenedores y cucharas atribuidos por esta guía a un restaurante en particular y el modelo ROJ tomará el valor de 1 si el color de esos tenedores o cucharas es rojo, de lo contrario será 0 .

Champérard describe el entorno y servicio de los restaurantes por el uso de un número de candelabros (VELAS) y azafates (AZAF), los cuales varían de 1 a 4 de acuerdo a, respectivamente, la calidad del entorno y el servicio.

\section{Otras variables}

- Restaurante nuevo

El modelo NUEVA indica que el restaurante aparece por primera vez en el manual GM (edición 2000). Con esta variable vemos si los restaurantes de referencia reciente son evaluados de igual manera que los restaurantes que entraron a la guía con anterioridad. 
- Historia de la gastronomía

Algunas veces, GM menciona si los restaurantes o chefs pertenecen a la historia de la gastronomía. Debido a que esta característica está conectada a influenciar en la nota, el modelo HIST tomará el valor de 1 cuando los comentarios incluyan remarcas tales como "este restaurante es un santuario para la tradición Landes"; de lo contrario será 0 .

\section{- El nombre del chef}

En la mayoría de los casos (81.1\%), los comentarios incluyen explícitamente el nombre del cocinero. Nosotros hemos decidido tomar esta disparidad en consideración con el modelo NOMBRE, que toma el valor de 1 cuando el nombre es mencionado; de lo contrario será 0 .

\section{PROCEDIMIENTO ESTIMATIVO}

Nuestra idea inicial fue establecer una regresión CATECORÍA contra CuisGM, un vector representando las 34 variables encontradas por Cuisine (ver subsección 2.1); $A M B$, un vector de dos variables incluyendo VINO y AMBGM; y $X$, un vector de variables exógenas (NOMBRE, HIST y NUEVA).

CATEGORIA $?=$ CuISCM??+AMB?? + X?? + u? $\quad ?=\mathrm{I}, \ldots \ldots \ldots, \mathrm{n}$.

Primero fue tentador reducir SET a su único y principal componente y CuisGM a un vector menos importante usando un programa componente principal. Pero los resultados obtenidos por CuisGM fueron desalentadores. Sin embargo, el principal componente para SET tomó en consideración cerca del $78 \%$ de la variación total. Por lo tanto, para un más fácil cómputo decidimos seguir el procedimiento con $\mathrm{AMB}$ como un indicador ${ }^{11}$.

En este procedimiento de cálculo, asumimos implícitamente que la dirección causal es del criterio del tipo de cocina al grado o nota y no lo contrario. En otras palabras, CuisCM es considerado como exógeno porque el criterio y los índices son determinados por el experto. Esto no es un supuesto fuerte si nos referimos al ejemplo del vino en el cual las notas están generalmente determinadas siguiendo el método de Davis, el cual consiste en ponderar cuatro criterios: examen visual $(12 \%)$, examen olfativo ( $24 \%)$, examen del gusto $(40 \%)$ y puntos generales $(24 \%)$.

Sin embargo, asumimos que las evaluaciones del entorno ( $A M B)$ son endógenas con respecto a CuisGM. En efecto, es muy probable que los chefs no escojan el entorno al azar, y podemos esperar ex ante una correlación significativa entre SET y CuisGM. Para evitar potenciales fallas asociadas con este típico problema endogenético y para obtener el verdadero efecto del entorno, usamos instrumentos para predecir AMB. Los instrumentos que hemos retenido son las evaluaciones dadas por Michelin (T\&C y ROJ) para el entorno. La razón principal para esta elección es que estas variables afectan estadísticamente al entorno pero no tienen efecto alguno (muy poco) en el criterio del tipo de cocina ${ }^{12}$.

Por el contrario, evaluaciones hechas por Champérard (VELAS y AZAF) han sido excluidas de la regresión por un significativo nivel de correlación con Cuis $\mathrm{GM}$. El cuadrante- $R$-obtenido para la regresión OLS ${ }^{13}$ de $A M B$ contra $T \& C$ y $R O J$ - es $0.257^{14}$. Para ver si OLS o 2SLS es el estimado apropiado aquí, aplicamos la prueba de Hausman (1978), la cual consiste en agregar una variable AMB -una predicción de SET gracias a la regresión auxiliar descrita arriba- a la ecuación de los expertos y probar si el parámetro correspondiente es significativo o no; esto es lo que llamamos la estadística Wu luego de Greene (2000, pp. 383-384). Una simple prueba de Wald indica que es significativo a un nivel del $5 \%(X 2$ calc $=4.02$ por $1 \mathrm{df}$.) y por lo tanto ese 2 SLS es el estimado apropiado de proceder ${ }^{15}$. Finalmente, debido a que las notas no son realmente continuas ( 11.5 no es una elección por ejemplo) sino ordenadas,

1 SET ha sido generada de la siguiente ecuación: 0.70711 VINO + 0.70711 AMBCM. SET es una variable ordenada dado que VINO y AMBGM sean ordenadas.

12 Esta conexión estadistica sugiere una convergencia general de las evaluaciones de los expertos en la vista del lugar. Probablemente se deba a que el entomo se evaluaa principalmente por un criterio objetivo como el número de meseros, etc.

13 De acuerdo con Angrist y Krueger (2001), "Usar una regresión linear para el primer estimado genera un segundo estimado consistente, aun con un modelo endógeno variable". Por lo tanto, no es necesario usar logit ordenados para generar valores predecibles para SET.

14 Aun si el cuadrante R es elevado (0.569) cuando la regresión incluye VELAS y AZAF.

15 Sin embargo, los resultados son comparables usando SET en vez de AMB y cuando AMB es adaptada de una regresión auxiliar incluyendo VELAS y AZAF en adición a T\&C y ROJ. 
llevamos la regresión siguiendo el método logit ordenado (Greene, 2000, pp. 875-879). Los resultados son presentados en la tabla I. Con un seudo R2 de 0.3756 , la calidad del ajuste es bastante satisfactoria para este tipo de regresión.

En la tabla II reportamos los resultados de pruebas $L R$ distintas para ver si los dos criterios principales en los cuales nos hemos centrado funcionan o no. Dos factores están detectados: el tipo de cocina es, afortunadamente, el más influyente, pero las notas de los expertos de GM también dependen del entorno (bodega, servicio y miembros del cuadro). Por lo tanto, los chefs no tienen una sino dos estrategias para aumentar en la jerarquía del estilo de cocina gastronómica.

\section{TABLA I.}

Estimación de los parámetros a través del MÉtodo de loGit ordenado para los factores QUe influYen EN LAS categorías de GaultMillau ${ }^{16}$

\begin{tabular}{|c|c|c|c|}
\hline Variables & Abreviaciones & Coeficiente & $z$-estad \\
\hline $\begin{array}{l}\text { Cocina } \\
\text { Comentarios generales } \\
\text { Número de superlativos } \\
\{1 ; 5] \\
{[5 ; 10[} \\
{[10 ; 15 \mid} \\
? 15\end{array}$ & $\begin{array}{l}\text { SUP1_5 } \\
\text { SUP5_10 } \\
\text { SUP10_15 } \\
\text { SUP_15 }\end{array}$ & $\begin{array}{l}0.78 \\
3.29 \\
5.34\end{array}$ & $\begin{array}{l}2.01 \mathrm{~b} \\
4.69^{\mathrm{a}} \\
4.09 \mathrm{a}\end{array}$ \\
\hline $\begin{array}{l}\text { Número de críticas generales } \\
\text { Estilo de cocina } \\
\text { No indicado } \\
\text { Solamente regional } \\
\text { Solamente clásica } \\
\text { Solamente creativa } \\
\text { Clásica y regional } \\
\text { Clásica y creativa } \\
\text { Regional y creativa } \\
\text { Clásica, regional y creativa }\end{array}$ & $\begin{array}{l}\text { CRIT } \\
\text { N/ESTILO } \\
\text { REG } \\
\text { CLAS } \\
\text { CREA } \\
\text { CLRE } \\
\text { CLCR } \\
\text { RECR } \\
\text { CLRECR }\end{array}$ & $\begin{array}{l}-0.36 \\
- \\
-0.46 \\
0.37 \\
0.65 \\
0.98 \\
0.68 \\
0.00 \\
1.86\end{array}$ & $\begin{array}{l}-0.71 \\
\\
-0.79 \\
0.69 \\
1.2 \\
1.61 \\
1.17 \\
0.001 \\
2.68 a\end{array}$ \\
\hline $\begin{array}{l}\text { Comentarios técnicos } \\
\text { Buena habil. técnic. (cocina, gusto...) } \\
\text { Critica de la técnica }\end{array}$ & $\begin{array}{l}\text { TECN+ } \\
\text { TECN- }\end{array}$ & $\begin{array}{l}1.63 \\
-0.05\end{array}$ & $\begin{array}{l}4.44 a \\
-0.1\end{array}$ \\
\hline $\begin{array}{l}\text { Comentarios varios } \\
\text { Combinación de sabores: } \\
\qquad \begin{array}{l}\text { Buena } \\
\text { Mala }\end{array} \\
\text { Refinarniento de los platos } \\
\text { Simplicidad de los platos } \\
\text { Diseño artistico de los platos } \\
\text { Sutileza de los platos }\end{array}$ & $\begin{array}{l}\text { SAB + } \\
\text { SAB- } \\
\text { REF } \\
\text { SIMPL } \\
\text { ART } \\
\text { SUT }\end{array}$ & $\begin{array}{l}0.94 \\
1.78 \\
1.21 \\
1.11 \\
0.14 \\
0.14\end{array}$ & $\begin{array}{l}2.08 b \\
2.12 b \\
1.96 b \\
2.49 a \\
0.15 \\
0.3\end{array}$ \\
\hline $\begin{array}{l}\text { Sofisticación: } \\
\text { Útil } \\
\text { Inútil }\end{array}$ & $\begin{array}{l}\text { SOF+ } \\
\text { SOF- }\end{array}$ & $\begin{array}{l}1.95 \\
-\end{array}$ & $\begin{array}{l}1.81 \mathrm{c} \\
-\end{array}$ \\
\hline $\begin{array}{l}\text { Generosidad de los platos } \\
\qquad \begin{array}{l}\text { Recalcada } \\
\text { Falta }\end{array} \\
\text { Menú rico (rico, diversificado...) }\end{array}$ & $\begin{array}{l}\text { GEN+ } \\
\text { GEN- } \\
\text { MENÚ }\end{array}$ & $\begin{array}{l}1.07 \\
-\end{array}$ & $1.77 \mathrm{c}$ \\
\hline
\end{tabular}




\begin{tabular}{|c|c|c|c|}
\hline $\begin{array}{l}\text { Ingredientes } \\
\text { Calidad excepcional } \\
\text { Mala calidad }\end{array}$ & $\begin{array}{l}\text { ING+ } \\
\text { ING- }\end{array}$ & $\begin{array}{l}-0.02 \\
0.73\end{array}$ & $\begin{array}{l}-0.05 \\
0.82\end{array}$ \\
\hline $\begin{array}{l}\text { Comestibles especiales: } \\
\text { Pescado (salmón, rodaballo,...) } \\
\text { Crustáceos } \\
\text { Caviar } \\
\text { Foie gras } \\
\text { Trufas } \\
\text { Queso }\end{array}$ & $\begin{array}{l}\text { PESC } \\
\text { CRUST } \\
\text { CAV } \\
\text { FOIE } \\
\text { TRUF } \\
\text { QUE }\end{array}$ & $\begin{array}{l}0.17 \\
-0.05 \\
1.02 \\
0.45 \\
0.72 \\
0.67\end{array}$ & $\begin{array}{l}0.52 \\
-0.14 \\
1.20 \\
1.25 \\
1.62 \\
0.68\end{array}$ \\
\hline $\begin{array}{l}\text { Ambiente, entorno (Cave, servicio y en- } \\
\text { torno) } \\
\text { Otras variables } \\
\text { Nueva referencia del restaurante } \\
\text { Historia de la gastronomía } \\
\text { Nombre del chef }\end{array}$ & $\begin{array}{l}\text { AMB } \\
\text { NUE } \\
\text { HIST } \\
\text { NOM }\end{array}$ & $\begin{array}{l}1.45 \\
-2.65 \\
1.22 \\
0.9\end{array}$ & $\begin{array}{l}5.02 a \\
-3.78 a \\
2.06 b \\
2.17 b\end{array}$ \\
\hline $\begin{array}{l}\text { Número de observaciones } \\
\text { ? } 2 \text { ( } 34 \mathrm{df} \text {.) } \\
\text { Log de probabilidades } \\
\text { Pseudo-R2 }\end{array}$ & & $\begin{array}{l}187 \\
268.77 \\
-223.39 \\
0.38\end{array}$ & \\
\hline $\begin{array}{l}\text { Parámetros umbrales } \\
12 \\
13 \\
14 \\
15 \\
16 \\
17 \\
18 \\
19\end{array}$ & & $\begin{array}{l}-2.69 \\
-0.91 \\
0.9 \\
3.26 \\
5.17 \\
8.73 \\
10.82 \\
12.74\end{array}$ & $\begin{array}{l}-3.58^{\mathrm{a}} \\
-1.53 \\
1.55 \\
5.11^{\mathrm{a}} \\
7.38^{\mathrm{a}} \\
9.66^{\mathrm{a}} \\
10.19^{\mathrm{a}} \\
9.89 \mathrm{a}\end{array}$ \\
\hline
\end{tabular}

a Diferente significativamente desde 0 a nivel de $1 \%$

b Diferente significativamente desde 0 a nivel de $5 \%$

c Diferente significativamente desde 0 a nivel de $10 \%$

Dándole una mirada más cercana a los detalles, sin sorprendemos, "vemos que los superlativos" (expresiones laudatorias más cursos apreciados) están positiva y significativamente conectados con la nota. Las evaluaciones también varían de acuerdo al estilo(s) de comida del chef. Diversificación es preferida en vez de especialización, como es sugerido con el coeficiente de CLRECR, lo cual es positivo y significante al nivel de $1 \%$. El manual GM parece apreciar, también, la técnica del chef. Buena habilidad técnica (TECN+) contribuye, ceteris paribus, a un mejoramiento significativo en la nota. Extrañamente, los críticos de la técnica (TECN-), así como los de la calidad de cursos (CRIT, TECN-, ING-), no tienen un impacto aparente en el nivel de la nota. En este contexto, los críticos parecen darle solo una advertencia a los chefs acerca de fallas en su tipo de cocina.

Los expertos de GM parecen tener una reacción positiva a las combinaciones de sabores $(\mathrm{SAB}+)$, manjares (REF), simplicidad (SIMPL) y positiva sofisticación en preparación (SOF+). Señales inesperadas son obtenidas para el coeficiente GEN $+y$ SAB-. La generosidad de los cursos actúa negativamente pero solo en un $10 \%$, mientras que una mala combinación de sabores tiene un impacto positivo en un grado de $5 \%$. Estos resultados probablemente se deban a que estos criterios no aparecen frecuentemente en los comentarios (observaciones 
15 y 9 respectivamente). La calidad de los ingredientes $y$ el uso de comidas raras no tienen influencia en la nota. Por otro lado un buen menú suele ser apreciado por los expertos de GM.

TABLA II: LA RATIO DEL LOG DE PROBABILIDADES PARA MEDIR EL GRADO DE SIGNIFICACIÓN DE DOS ESTRATEGIAS ALTERNATIVAS

\begin{tabular}{|l|l|}
\hline $\begin{array}{l}\text { Regresión completa } \\
\text { Valor del Log de probabilidades }\end{array}$ & -223.39 \\
\hline $\begin{array}{l}\text { No cocina } \\
\text { Valor del Log de probabilidades } \\
\text { ?2 test (33 df.) } \\
\text { ?2 Valor tabulada (nivel 5\%) }\end{array}$ & -336.4 \\
& 226.03 \\
\hline No ambiente/entorno & 47.39 \\
Valor del Log de probabilidades & -236.88 \\
?2 test (1 df.) & 26.98 \\
?2 Valor tabulada (nivel 5\%) & 3.84 \\
\hline
\end{tabular}

Finalmente, otros elementos (incluidos en el vector $X$ ) discriminan entre las notas bajas y altas. Primero, los integrantes nuevos (NUE) obtienen, ceteris paribus, una nota baja como si los expertos de GM esperasen que se consoliden en el futuro. Segundo, GM recompensa el tipo de cocina que es "personalizado", como se indica por el nivel de significado obtenido por NOMBRE, cuando el nombre del chef es mencionado en el comentario. Finalmente, para un cocinero o un restaurante, ser parte de la historia del tipo de cocina gastronómica tiene un impacto positivo en las notas de CM.

\section{CONCLUSIONES}

De acuerdo con GM, solo el tipo de cocina es tomado en cuenta cuando se evalúan los restaurantes: "rangos del 11 al 19 solo evalúan la calidad del tipo de cocina" (Manual GM, 2000, p. 42). Nuestros resultados sugieren lo contrario: los chefs tienen dos estrategias para obtener una buena nota en esta guía, una del tipo de cocina y otra del entorno. Cabe mencionar que el primero prevalece sobre el segundo.
En el contexto de la primera estrategia, el knowhow y la creatividad del chef son elementos cruciales, mientras que en el contexto de la segunda estrategia es solo su capacidad de invertir en el entorno $y$ en el servicio los que cuentan. El entorno (bodega, servicio y miembros del cuadro) obviamente tiene un peso, aunque menor que el arte de cocinar (las habilidades técnicas, combinación de sabores, variedad en estilos de cocina, menús atractivos, la delicadeza, sofisticación y simplicidad de sus preparaciones). Estos resultados son similares a los de Gergaud y Vignes (2000) para el mercado de champagne, donde mostraron que las firmas invierten en reputación produciendo un vino de alta calidad o mediante el reconocimiento público hecho a través de la publicidad.

Este resultado puede explicar por qué en años recientes los chefs han tenido la tendencia de invertir cada vez más en el entorno. Existen muchos ejemplos ilustrativos: Georges Blanc (23 millones de euros invertidos en Vonnas), Marc Veyrat ( 10 millones de euros invertidos en Annecy y Megéve), Bernard Loiseau ( 8 millones de euros invertidos en Saulieu). El caso más extremo es tal vez el de Pierre Gagnaire en Saint Etienne; él fue forzado a cesar su negocio debido a deudas demasiado elevadas.

En este contexto paradójico, un restaurante que ofrece cocina de primer nivel no llegará a ser top si es que sus empleados no tienen una actitud profesional, si la bodega no se encuentra bien abastecida, o si los manteles están manchados y no planchados. Pero un chef que invierte solo en el entorno y la atmósfera dejando de lado su tipo de cocina, tendrá pocas chances de ser reconocido como cocinero de alta clase por los expertos o por sus compañeros. En términos económicos, se puede sugerir que una estrategia mixta es más eficiente que una pura ${ }^{17}$. Todo esto indica que la relación entre estas dos estrategias no es lineal: el beneficio de estrategias no alimentarias probablemente dependa del nivel al cual el chef aspire. Un segundo paso en este análisis sería verificar este punto estimando una función de producción Translog para ver si las dos estrategias son sustitutas o complementarias. 


\section{AGRADECIMIENTOS}

Muy honrados por haber sido premiados con el President's Prize en Rotterdam 2002, durante la ACEI Conference, los autores agradecen a los "gurús" del Comité Científico por esa decisión.
También queremos expresar nuestros agradecimientos a C. Barrère, T. Coupé, S. Creigh-Tyte, V. Ginsburgh, J.M. Schuster y a los árbitros anónimos por sus útiles comentarios. Los autores son los únicos responsables por los errores contenidos en el artículo.

\section{APENDICE: EstadíSTICAS desCRIPTIVAS}

\section{TABLA A-1}

\begin{tabular}{|c|c|c|c|c|}
\hline VARIABLES & ABREVIACIONES & MODALIDADES & \multicolumn{2}{|c|}{$\begin{array}{l}\text { ESTADÍTICAS } \\
\text { DESCRIPTIVAS }\end{array}$} \\
\hline $\begin{array}{l}\text { Categoría de expertos } \\
\text { (Gaul(Millau) }\end{array}$ & Categoría & Continuos & \multicolumn{2}{|c|}{$\begin{array}{l}\text { Mediana }=14.88 \\
\text { Desv. estándar }=1.72 \\
\text { Min }=11 ; \operatorname{Max}=19\end{array}$} \\
\hline $\begin{array}{l}\text { Cocina } \\
\\
\text { Número de superlativos } \\
{[1 ; 5 \mid} \\
|5 ; 10| \\
|10 ; 15| \\
? 15\end{array}$ & $\begin{array}{l}\text { SUP1_5 } \\
\text { SUP5_10 } \\
\text { SUP10_15 } \\
\text { SUP_15 }\end{array}$ & $\begin{array}{l}S i=1 ; N o=0 \\
S i=1 ; N o=0 \\
S i=1 ; N o=0 \\
S i=1 ; N o=0\end{array}$ & $\begin{array}{l}32.97 \% \\
50.27 \% \\
12.43 \% \\
4.32 \%\end{array}$ & $\begin{array}{l}\text { ( } 61 \text { obs) } \\
\text { (93 obs) } \\
(23 \text { obs) } \\
\text { (8 obs) }\end{array}$ \\
\hline $\begin{array}{l}\text { Número de críticas generales } \\
\text { Estilo de cocina } \\
\text { No indicado } \\
\text { Solamente regional } \\
\text { Solamente clásica } \\
\text { Solamente creativa } \\
\text { Clásica y regional } \\
\text { Clásica y creativa } \\
\text { Regional y creativa } \\
\text { Clásica, regional y creativa }\end{array}$ & $\begin{array}{l}\text { CRIT } \\
\text { N/ESTILO } \\
\text { REC } \\
\text { CLAS } \\
\text { CREA } \\
\text { CLRE } \\
\text { CLCR } \\
\text { RECR } \\
\text { CLRECR }\end{array}$ & $\begin{array}{l}\mathrm{Si}=1 ; \mathrm{No}=0 \\
\mathrm{Si}=1 ; \mathrm{No}=0 \\
\mathrm{Si}=1 ; \mathrm{No}=0 \\
\mathrm{Si}=1 ; \mathrm{No}=0 \\
\mathrm{Si}=1 ; \mathrm{No}=0 \\
\mathrm{Si}=1 ; \mathrm{No}=0 \\
\mathrm{Si}=1 ; \mathrm{No}=0 \\
\mathrm{Si}=1 ; \mathrm{No}=0 \\
\mathrm{Si}=1 ; \mathrm{No}=0\end{array}$ & $\begin{array}{l}16.76 \% \\
20,54 \% \\
9.19 \% \\
14.05 \% \\
15.68 \% \\
10.27 \% \\
14.05 \% \\
8.65 \% \\
7.57 \%\end{array}$ & $\begin{array}{l}(31 \text { obs) } \\
(38 \text { obs) } \\
(17 \text { obs) } \\
(26 \text { obs }) \\
(29 \text { obs) } \\
(19 \text { obs) } \\
(26 \text { obs }) \\
(16 \text { obs) } \\
(14 \text { obs) }\end{array}$ \\
\hline $\begin{array}{l}\text { Comentarios técnicos } \\
\text { Buena habil. técnic. (cocina, gusto...) } \\
\text { Crítica de la técnica }\end{array}$ & $\begin{array}{l}\text { TECN+ } \\
\text { TECN- }\end{array}$ & $\begin{array}{l}\mathrm{Si}=1 ; \mathrm{No}=0 \\
\mathrm{Si}=1 ; \mathrm{No}=0\end{array}$ & $\begin{array}{l}57.84 \% \\
14.59 \%\end{array}$ & $\begin{array}{l}\text { (107 obs) } \\
(27 \text { obs) }\end{array}$ \\
\hline $\begin{array}{l}\text { Comentarios varios } \\
\text { Combinación de sabores: } \\
\text { Buena } \\
\text { Mala } \\
\text { Refinamiento de los platos } \\
\text { Simplicidad de los platos }\end{array}$ & $\begin{array}{l}\text { SAB+ } \\
\text { SAB- } \\
\text { REF } \\
\text { SIMPL }\end{array}$ & $\begin{array}{l}S i=1 ; N o=0 \\
S i=1 ; N o=0 \\
S i=1 ; N o=0 \\
S i=1 ; N o=0\end{array}$ & $\begin{array}{l}24.32 \% \\
4.86 \% \\
7.57 \% \\
20.00 \%\end{array}$ & $\begin{array}{l}\text { (45 obs) } \\
\text { (9 obs) } \\
(14 \text { obs) } \\
(37 \text { obs) }\end{array}$ \\
\hline
\end{tabular}




\begin{tabular}{|c|c|c|c|c|}
\hline $\begin{array}{l}\text { Diseño artístico de los platos } \\
\text { Sutileza de los platos }\end{array}$ & $\begin{array}{l}\text { ART } \\
\text { SUT }\end{array}$ & $\begin{array}{l}S i=1 ; N o=0 \\
S i=1 ; N o=0\end{array}$ & $\begin{array}{l}4.86 \% \\
11.89 \%\end{array}$ & $\begin{array}{l}\text { ( } 9 \text { obs }) \\
(22 \text { obs })\end{array}$ \\
\hline \multicolumn{5}{|l|}{ Sofisticación: } \\
\hline Útil & SOF + & $\mathrm{Si}=1 ; \mathrm{No}_{0}=0$ & $3.24 \%$ & (6 obs) \\
\hline \multicolumn{5}{|l|}{ Generosidad de los platos } \\
\hline Recalcada & GEN+ & $\mathrm{Si}=1 ; \mathrm{No}=0$ & $8.11 \%$ & (15 obs) \\
\hline Falta & GEN- & $\mathrm{Si}=1 ; \mathrm{No}=0$ & $1.08 \%$ & (2 obs) \\
\hline Menú rico (rico, diversificado...) & MENÚ & $\mathrm{Si}=1 ; \mathrm{No}=0$ & $15.68 \%$ & (29 obs) \\
\hline \multicolumn{5}{|l|}{ Ingredientes } \\
\hline Calidad excepcional & INC+ & $\mathrm{Si}=1 ; \mathrm{No}=0$ & $40.00 \%$ & (74 obs) \\
\hline Mala calidad & ING. & $\mathrm{Si}=1 ; \mathrm{No}=0$ & $3.24 \%$ & (6 obs) \\
\hline \multicolumn{5}{|l|}{ Comestibles especiales: } \\
\hline Pescado (salmón, rodabalio,...) & PESC & $\mathrm{Si}=1 ; \mathrm{No}=0$ & $55.14 \%$ & (102 obs) \\
\hline Crustáceos & CRUST & $\mathrm{Si}=1 ; \mathrm{No}_{\mathrm{O}}=0$ & $46.49 \%$ & (86 obs) \\
\hline Caviar & CAV & $\mathrm{Si}=1 ; \mathrm{No}=0$ & $4.86 \%$ & (9 obs) \\
\hline Foie gras & FOIE & $\mathrm{Si}=1 ; \mathrm{No}=0$ & $33.51 \%$ & (62 obs) \\
\hline Trufas & TRUF & $\mathrm{Si}=1 ; \mathrm{No}=0$ & $17.03 \%$ & (32 obs) \\
\hline Queso & QUE & $\mathrm{Si}=1 ; \mathrm{No}=0$ & $3.24 \%$ & (6 obs) \\
\hline \multicolumn{5}{|l|}{ Ambiente y servicio } \\
\hline \multicolumn{5}{|l|}{$\begin{array}{l}\text { Michelin: } \\
\text { Número de tenedores/cucharas }\end{array}$} \\
\hline Número de tenedores/cucharas & $\mathrm{T} \& \mathrm{C}$ & Escala cardinal & \multirow{2}{*}{\multicolumn{2}{|c|}{$\begin{array}{l}\text { Med }=2.97 \\
\text { Dev.Est. }=0.94 \\
\text { Min. }=1 ; \text { Máx. }=3 \\
25.95 \% \text { (48 obs) }\end{array}$}} \\
\hline Tenedores rojos/cucharas & $\mathrm{ROJ}$ & $\mathrm{Si}=1 ; \mathrm{No}=0$ & & \\
\hline \multirow[t]{2}{*}{ Gaultumillau } & AMBCM & Escala cardinal & \multirow{2}{*}{\multicolumn{2}{|c|}{$\begin{array}{l}\text { Med }=2.15 \\
\text { DevEst. }=0.38 \\
\text { Min. }=1 ; \text { Máx. }=3\end{array}$}} \\
\hline & & & & \\
\hline \multicolumn{5}{|l|}{$\begin{array}{l}\text { Champérard: } \\
\text { Número de velas }\end{array}$} \\
\hline & & & \multicolumn{2}{|c|}{$\begin{array}{l}\text { Med }=2.47 \\
\text { DevEst. }=0.68 \\
\text { Mín. }=.1 ; \text { Máx. }=4\end{array}$} \\
\hline Número de azafates & AZAF & Escala cardinal & \multicolumn{2}{|c|}{$\begin{array}{l}\text { Med }=2.40 \\
\text { DevEst. }=0.64 \\
\text { Min. }=1 ; \text { Máx. }=4\end{array}$} \\
\hline \multirow{4}{*}{$\begin{array}{l}\text { Otras variables } \\
\text { Restaurantes/nueva referencia } \\
\text { Historia de gastronomía } \\
\text { Nombre del chef }\end{array}$} & & & & \\
\hline & NUE & $\mathrm{Si}=1 ; \mathrm{No}=0$ & $4.86 \%$ & (9 obs) \\
\hline & HIST & $\mathrm{Si}=1 ; \mathrm{No}=0$ & $9.19 \%$ & (17 obs) \\
\hline & NOMBRE & $\mathrm{Si}=1 ; \mathrm{No}=0$ & $81.08 \%$ & (150 obs) \\
\hline
\end{tabular}




\section{REFERENCIAS BIBLIOGRÁFICAS}

Albrecht, J., Lang, H. and Vroman, S. (2002). "The Effect of Information on the Well-Being of the Uninformed: What's the Chance of Getting a Decent Meal in an Unfamiliar City?". International Journal of Industrial Organization 20 (2): 139-162.

Angrist, J.D. and Krueger, A.B. (2001). "Instrumental Variables and the Search for Identification: From Supply and Demand to Natural Experiments". Journal of Economic Perspectives 15 (4): 69-85.

Ashenfelter, O. and Jones, G. (2000). "The Demand for Expert Opinion: Bordeaux Wine". Cahier Scientifique de l'Observatoire des Conjonctures Vinicoles Européennes 3.

Banerjee, A. V. (1992). "A Simple Model of Herd Behavior". Quarterly Journal of Economics 107 (3): 797-817.

Becker, G.S. (1991). "A Note on Restaurant Pricing and Other Examples of Social Influences on Price". Journal of Political Economy 99 (5): $1109-1116$.

Card, D. (1999). "The Causal Effect of Education on Earnings", in O. Ashenfelter and D. Card (eds.). Handbook of Labor Economics. North Holland, Amsterdam, pp. 1801-1863.

Chossat, V. (2001). Les processus de sélection sur un marché, le cas de la grande cuisine. These pour le Doctorat en Sciences Economiques, Université de Reims Champagne - Ardenne.

Darby, M. and Karni, E. (1973). "Free Competition and the Optimal Amount of Fraud". Journal of Law and Economics 16.

Favereau, O. and Lazega, E. (2002). Conventions and Structures in Economic Organization: Markets, Networks and Hierarchies. Edward Elgar Press.
Gergaud, O. and Vignes, A (2000). "Emergence et dynamique du phénomene de réputation. Le vin de Champagne: entre savoir-faire et faire savoir". Revue d'Economie Industrielle 91 (1): $55-74$

Ginsburgh, V. (2002). "Award, Success and Aesthetic Quality in the Arts". Manuscript, ECARES, Université Libre de Bruxelles.

Ginsburgh, V. and Van Ours, J.C. (2002). "Expert Opinion and Compensation: Evidence from a Musical Competition". American Economic Review, forthcoming.

Greene, W.H. (2000). Econometric Analysis; 4th edn. Prentíce Hall International Editions.

Hausman, J.A. (1978). "Specification Tests in Econometrics". Econometrica 46: 1254-1270.

Institut National de la Statistique et des Etudes Economiques (1999). La France des Services, Département des activités tertiaires.

Lancaster, K.J. (1966). "A New Approach to Consumer Theory". Journal of Polítical Economy 74: 132-157.

Nelson, P. (1970). "Information. and Consumer Behavior". Journal of Political Economy 78 : 311-329.

(1974) "Advertising as Information". Journal of Political Economy 82: 728-754.

Wijnberg, N.M. (1995). "Se1ection Processes and Appropriability in Art, Science and Technology". Journal of Cultural Economics 19 (3): 221-235. 